\title{
DETERMINING PERCENTAGE OF BROKEN RICE BY USING IMAGE ANALYSIS
}

\author{
H. Aghayeghazvini ${ }^{1, *}$, A. Afzal ${ }^{1}$, M. Heidarisoltanabadi ${ }^{1}$, S. Malek ${ }^{1}$, \\ L. Mollabashi ${ }^{2}$ \\ 1 Esfahan Agricultural Research Center, Engineering Department, Esfahan, IRAN \\ 2 Esfahan University of Technology, Faculty of Physics, Esfahan, IRAN \\ * Corresponding author, Address: Esfahan Agricultural Research Center, Engineering \\ Department, post box 81785-199 Esfahan, IRAN, Tel: +98-311-7760061,Fax: +98-311- \\ 7757022, Email: Aghayeghazvini@yahoo.com
}

Abstract: During the rice milling process, a huller system is used to remove rough rice hull, then abrasive or frictional mill product whitened rice. In such machines adjustment and control, operation is important, because breakage percentage of rice is one main factor in determination of quality. Today's image processing techniques has become an increasingly popular and cost-effective method. The estimation of rice quality by image processing as a tool to determine broken percentage is the aim of this study. The images were acquired by a digital camera (1944 x 2592 pixels) from grains spread on corrugated plate, and then analyzed by scion software. Statistical analysis showed a significant correlation (greater than 0.9 ) between the results obtained from the proposed method and conventional method. This work indicates that the digital processing technique can be used for estimating broken grains.

Keywords: milling process, digital image processing, whitened rice, digital camera, conventional method.

\section{INTRODUCTION}

Rice is the dominant staple food crop in developing countries. One of the most important aspects of rice grain is its milling process. The control of percentage of broken kernels and whiteness (degree of milling) in milled rice is required to minimize the economic loss in rice milling.

Please use the following format when citing this chapter:

Aghayeghazvini, H., Afzal, A., Heidarisoltanabadi, M., Malek, S. and Mollabashi, L., 2009, in IFIP International Federation for Information Processing, Volume 294, Computer and Computing Technologies in Agriculture II, Volume 2, eds. D. Li, Z. Chunjiang, (Boston: Springer), pp. 1019-1027. 
Milling of rough rice (or paddy) is usually done at about $12-14 \%$ wet basis moisture content to produce white, polished edible grain, due to consumer preference. From the economic point of view, the quality of milled rice and its control are of paramount importance since the grain size and shape, whiteness, broken percentage and cleanliness are strongly correlated with the transaction price of rice (Conway et al., 1991). All these factors are closely related to the process of milling, in which rough rice is first subjected to dehusking or removal of hulls and then to the removal of brownish outer bran layer, known as whitening. Finally, polishing is carried out to remove the bran particles providing surface gloss to the edible white portion. A high percentage of broken grains in the milled product or low head rice recovery represent a direct economic loss to the millers.

The degree of milling determines the extent of removal of bran layer from the surface of milled kernels and thus it is very important to measure percentage of broken rice. Hence, in the milling process, the pressure in the milling chamber and the duration of milling must be adjusted to get the maximum output.

The extent of losses during milling depends on many factors, such as variety and condition of rough rice, degree of milling required, the kind of rice miller used, and the operators. Besides rubber roll dehuskers, two types of milling machines, namely, abrasive and frictional types are used for whitening and polishing of grain, respectively. In both types of machines, the degree of milling is also controlled by adjusting the pressure in the milling chamber by means of a spring-loaded counterweight at the discharge outlet. In practice, most control systems for rice milling equipment are essentially based on manual operation (Yadav et al., 2001). Informal contacts with several commercial mills in Esfahan province in central Iran revealed that milled rice quality is regularly monitored manually and visually at approximate time intervals about $1 \mathrm{~h}$ due to unavailability of continuous online measurement methods. Actual determination of broken percentage, grading, head rice yield (HRY) and milled degree of milling are made by laboratory measurements. The necessary adjustments made by a trained operator, based on visual inspection and the results of laboratory measurements, take effect in a few minutes to produce milled rice with a minimum amount of broken kernels and maximum degree of kernel whiteness. Usually milled rice samples obtained by milling test in the laboratory are supplied to the operator and used as reference for each grade of the degree of milling.

Despite the extensive use of image analysis in manufacturing and medical industries, its applications are almost low existent in grain-based industries. The determination of milled rice quality parameters by image processing techniques will enable regular monitoring of milling operation in an 
objective manner, and thus allow the operator to quickly react within a few minutes to changes in material properties.

Yadav et al., (2001) used digital image analysis to determine the (HRY), representing the proportion by weighting milled kernels with three quarters or more of their original length, and the whiteness of milled rice on ten varieties of Thai rice. They reported that in case of the whiteness of milled samples, the values provided by a commercial whiteness meter and the mean of gray level distribution determined by image analysis correlated with an R2 value of 0.99 .

Fant et al. (1994) determined the gray scale intensity in the digital images of rice samples subjected to various degrees of milling and correlated the mean gray level with lipids concentration on the surface of rice kernels. Liu et al. (1998) used digital image analysis to estimate the area of the bran layer on the surface of rice kernels and correlated with the surface lipids concentration determined by chemical analysis. They reported that the degree of milling could be measured quickly and accurately in terms of the surface lipids concentration in a milled rice sample.

The main objective of the present study was to develop quick and practical techniques that could be used for estimating percentage of broken rice and rice grading based on two-dimensional imaging of milled rice kernels being sampled at regular intervals.

\section{APPROACH TO THE PROBLEM}

Percentage of broken rice could be expressed relative to the weight of broken rice rather than total weight of milled rice in a simple. In general, it is possible to estimate the weight of the objects having regular shapes based on their dimensional characteristics. Therefore, simple models could be developed for estimating the percentage of broken rice and grading of the milled rice samples using image analysis from the measurements of dimensional features and gray level distribution, respectively, as described in the following sections.

Based on the results of an earlier study for a single variety of rice (Yadav and Jindal, 1998), it was hypothesized that the weight of individual kernels, whether of head rice and broken fraction, is proportional to their respective dimensional features. Accordingly, the weight of a milled rice sample might be expressed as a power function of a composite characteristic dimensional feature derived from the whole and broken kernels in that sample as described in the following sections.

On the whole, the characteristic dimensional features of the kernels could be their length (L), thickness (T) and area (A) based on individual measurements. The weight of rice in the image of a milled rice sample is: 


$$
\mathrm{W}=\rho \cdot \mathrm{T} \cdot \mathrm{A}
$$

Where $\mathrm{W}$ is the weight of rice in a milled rice sample, $\rho$ is the density of sample, $\mathrm{T}$ is the mean of grain thickness and $\mathrm{A}$ is the projected area of rice in an image. Generally, thickness and density are two constant parameters in a milled rice sample.

\section{MATERIALS AND METHODS}

\subsection{Rice Samples}

Milled rice samples of one variety, namely, Sazandegi were obtained from the four commercial mills in Esfahan, with moisture contents about $12 \%$ dry basis. Four samples of each mill weighting individually about $250 \mathrm{~g}$ were taken from each mill and kept separately in polyethylene bags. Then four $20 \mathrm{~g}$ samples were obtained from each bag. Thus, 16 samples were used for determining the milled rice characteristics.

\subsection{Imaging of Rice Grains}

A diagram of the imaging system is shown in Fig. 1. It consisted of a lighting unit, a color CCD camera connected to a host Centrino 2GH Toshiba computer. The lighting unit comprised with four circular $32 \mathrm{~W}$ fluorescent lamps but under indoor ambient day light condition. The camera (model DSC-T7, Sony Inc., Japan) was equipped with a zoom lens (model VarioTessar 3x, F 3.5 and 38-114 mm zoom, Carl ziss Inc., Germany) capable of producing image output with resolution of (1944 x 2592 pixels). The operation of the system was carefully controlled for extracting reproducible features from the captured i Determining Percentage of Broken Rice by Using Image Analysis

mages of milled rice samples through various adjustments. All the captured frames were 8-bit (0-255) gray scale images. Each sample of rice kernels was spreaded manually without touching each other on a background about $100 \times 70 \mathrm{~cm}$ areas directly under the CCD camera. The dimensional features of individual rice images were extracted using the Scion software version 4.0.3.2 developed at Scion Corporation. The imaging system was calibrated with the help of a triangular shape cardboard having dimensions 46.6-34.9-31.4 mm. The linear measurements on rice grains dimensions 
were subsequently converted into actual values based on the calibration results.

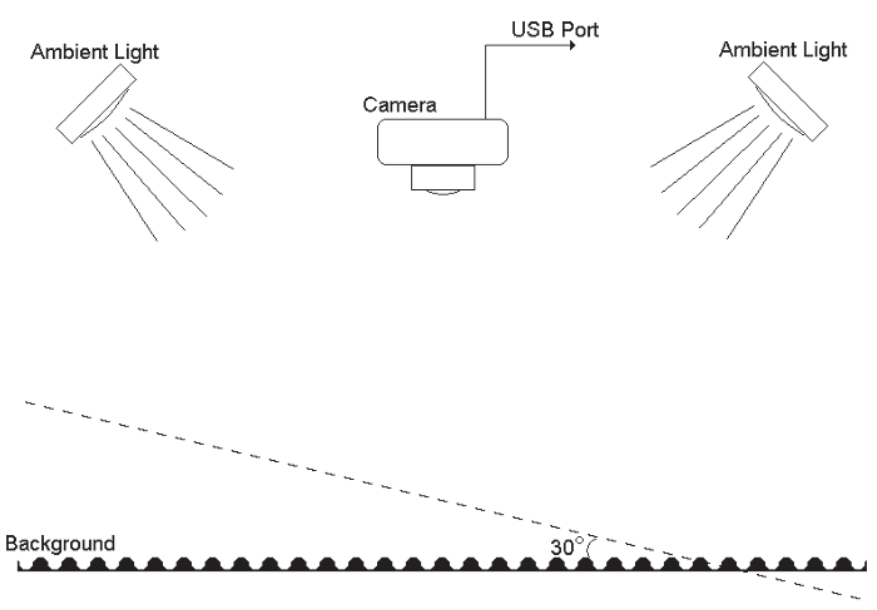

Fig. 1. Schematic diagram of the image analysis system.

\subsection{Determination of Broken Rice Percentage}

A laboratory rice grader (model TRG 05A, Stake Engineering Co. Ltd., Japan) was used to separate the head and broken kernels in milled rice samples through appropriate adjustment of its settings. The Broken Rice precentag was based on the kernel length equal to or more than $75 \%$ of the average length of whole whitened rice kernels, as already defined. It was found that the grader required a minimum of $12 \mathrm{~g}$ milled rice for obtaining reproducible values of broken rice percentage differing by less than $1 \%$.

Therefore, representative samples of milled rice weighing $20 \mathrm{~g}$ were obtained with a specially fabricated sample divider and separated into head and broken fractions by the laboratory grader for determining broken rice percentage. The use of small test samples was necessary to limit the total number of rice kernels for subsequent image analysis. In view of the imprecise separation of kernels by the laboratory grader, all head and broken kernels in their respective fractions were separated manually with the assistance of imaging system and weighed to compute the actual broken rice percentage of the representative sample. Later all kernels in the head and broken rice fractions were imaged to extract their dimensional features, namely, length (L), mean thickness (D) and area (A). The values of estimated dimensions were then computed for different milled rice samples and related with their actual broken rice percentage. Finally, the broken rice percentage obtained from the laboratory grader was compared with the actual broken rice percentage estimated by image analysis. 


\subsection{Results and Discussion}

Use of corrugated surface prevented the overlap of grains spread on it and grains were randomly positioned on the surface with different angles and by their length. This resulted to use the thickness average of the grains in the calculations. Dimensional features of the rice kernels were extracted from their respective images by separating them from the background and identifying each image with a unique number with the help of the Scion image software. Fig. 2 shows typical images of the numbered rice kernels used for the measurement of characteristic dimensional features and the computation of broken rice percentage for any selected rice sample. Figs. 3-5 present the relationships between estimated and actual based on kernel length and area, respectively. Regression analysis showed the existence of a power-law relationship between estimated and actual data. Table 2 presents the values of parameters estimated by regression function of SPSS version 9.05 for Windows with $95 \%$ confidence interval in each case. These results validated the hypothesis that the broken rice percentage of milled rice sample could be estimated with root mean square error (RMSE) of less than $2 \%$ from the dimensional features extracted from the images of the rice kernels in their natural rest position.

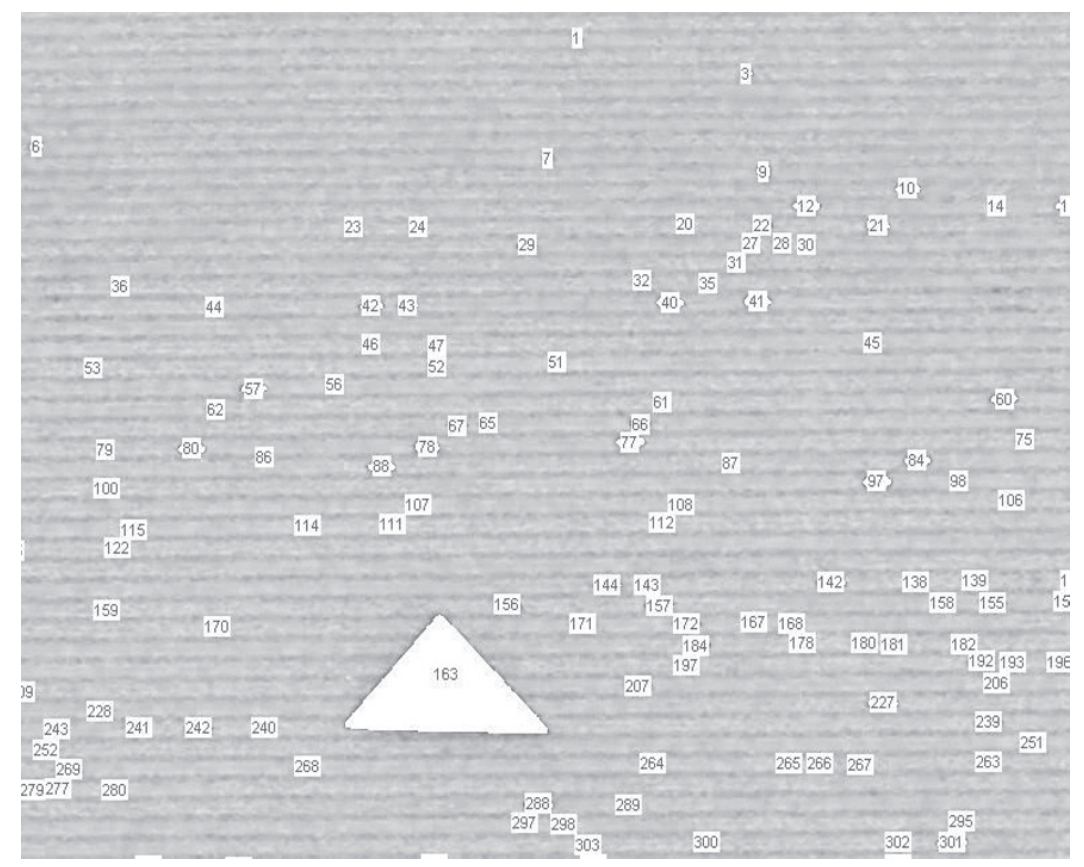

Fig. 2. An image of rice sample. 
The area of kernel images provided the best estimation of broken rice percentage in terms of the RMSE determined from the actual and estimated values for all samples.

The separation of milled rice samples by the laboratory grader often resulted in overlapping head and broken rice fractions. Therefore, the broken rice percentage obtained from the laboratory grader was compared with the broken rice percentage estimation based on the measurement of area of kernels as shown in Fig. 1. These results confirmed the discrepancies encountered in the determination of HRY by the laboratory grader in comparison with manual inspection of kernels by image analysis. These differences in HRY were possibly due to the imprecise separation of the whole and broken kernels by the laboratory grader than the more accurate vision-based measurements.

The HRY determination by the laboratory grader was influenced by its operating settings such as the size of indentation in the rotating cylinder and the inclination angle of the receiving trough. In this study, the operating conditions of the grader were identical for all rice varieties. Also, the proportion of the overlap between the head and broken fractions depended upon the changes in the dimensional characteristics of the rice kernels during the milling operation and the differences in rice varieties.

These results further implied that the percentage of broken rice estimated and thus grading by image analysis and the laboratory grader were indeed related, and could be used for monitoring the milling operation of different rice varieties.

Table 1. Result of regression analysis for estimating percentage of broken rice from dimensional characteristics of rice grain images.

\begin{tabular}{lccc}
\hline Characteristic dimension & Regression parameters & R2 & RMSE (\%) \\
\hline Length & 1.06 & 0.93 & 0.7 \\
Percentage of broken rice & 1.07 & 0.92 & 1.75 \\
\hline
\end{tabular}

The developments of such low-cost machine vision-based techniques that either enhance or replace currently used manual methods may pave the way for rapid assessment, and thus better control of rice milling operations in a conventional setting

It seems likely that similar procedures could be adopted for the inspection of some other crop quality, based on the techniques described in this paper. 


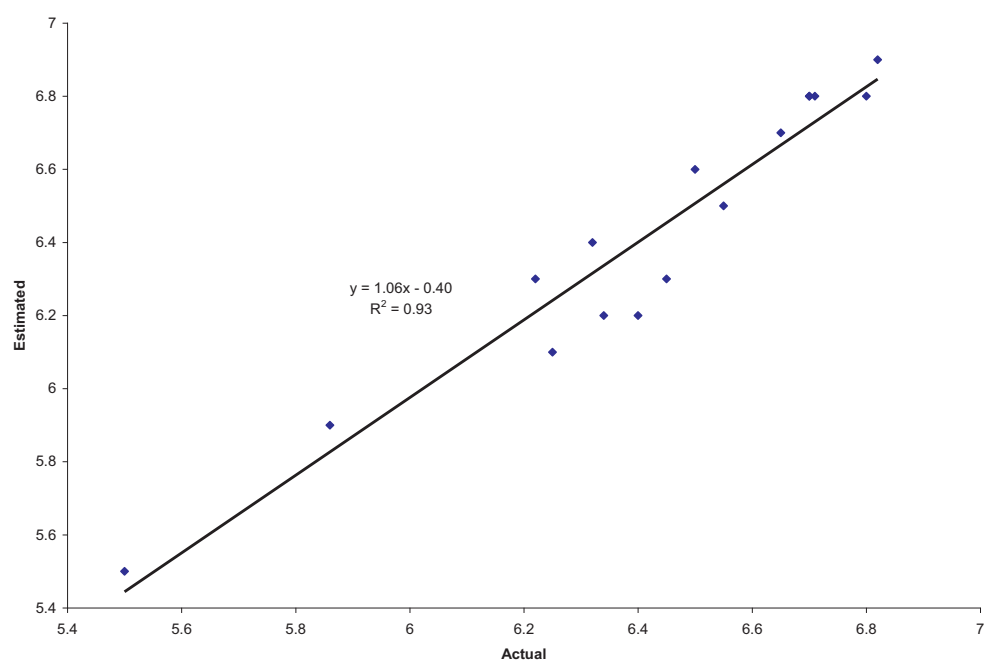

Fig. 3. Comparison of actual and estimated length of rice grains.

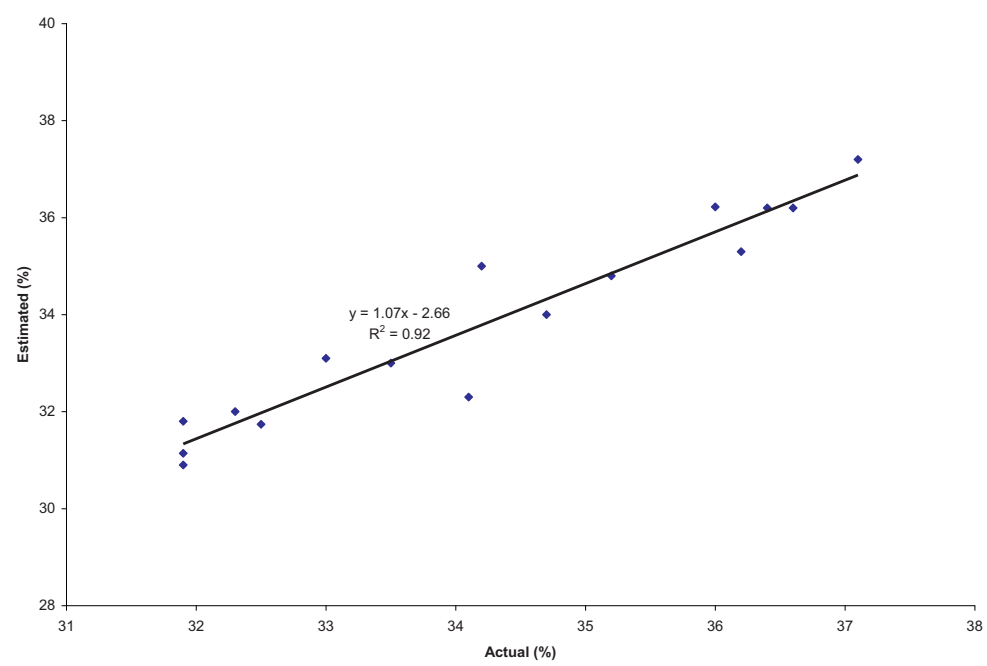

Fig. 4 Comparison of actual and estimated percentage of broken rice.

\section{CONCLUSIONS}

Results showed that this project could be used practically for optimizing the controls in mills and rice buying centers, furthermore showed that broken rice percentage and grading of milled rice could be estimated from two-dimensional images of the milled rice kernels in their natural rest 
position. The broken rice percentage showed a distinct power-law relationship with the characteristic dimension ratio defined in terms of length and area of head and broken rice kernels. The developed empirical relationships for estimating broken rice percentage and milled rice grading could be used for regular monitoring and better control of rice milling operations. More study is required to determine the possible use of this research for other cereals.

\section{ACKNOWLEDGEMENTS}

The authors would like to thank the Mr. B.k. Yadav and V.K. Jindal .

\section{REFERENCES}

Blasco, J., Alexios, N. and Molto, E. Machine vision system for automatic quality grading of fruit. Biosystem Eng, 2003, 85(4): 415-423.

Forbe, K. Volume estimation of fruit from digital profile image. M. Sc. Thesis of Electronic Engineering. Cape Town University. Cape Town. South Africa, 2000.

Gonzalez, R.C. and Wood R.E Digital image processing. Second edition. Prentice Hall press.New Jersey. USA, 2001.

Yadav, B.K., Jindal, V.K Monitoring milled rice characteristics by image analysis. In: Salokhe, V. M., Jianxia, Z, 1998, (Eds.), Proceedings of the International Agricultural Engineering Conference, Bangkok, Thailand, 7-10 December, pp. 963-971.

Yadav, B.K., Jindal, V.K. Monitoring milling quality of rice by image analysis. Elsevier.Computers and Electronics in Agriculture, 2001, 33:19-33. 